\title{
Plasmonlike resonances in atomic chains: A time-dependent density-functional theory study
}

\author{
Yu-Hui Huang, ${ }^{1}$ Ken-Ming Lin, ${ }^{1, *}$ T. C. Leung, ${ }^{1, \dagger}$ and C. T. Chan ${ }^{2}$ \\ ${ }^{1}$ Department of Physics, National Chung Cheng University, Chiayi 621, Taiwan, Republic of China \\ ${ }^{2}$ Department of Physics, The Hong Kong University of Science and Technology, Hong Kong, People's Republic of China
}

(Received 28 November 2013; revised manuscript received 28 July 2014; published 20 August 2014)

\begin{abstract}
We studied plasmonlike resonances in one-dimensional (1D) atomic chain systems by using time-dependent density-functional theory (TDDFT) and local density functional theory. Recent TDDFT studies have shown the coexistence of longitudinal and transverses collective plasmonlike resonances in the atomic chains of simple and noble metals. Such atomic chains contain only a few atoms. The induced polarization occurs along the atomic chain in longitudinal mode and perpendicular to the atomic chain in transverse mode. To understand the emergence of plasmonlike resonance in $1 \mathrm{D}$ atomic chains better, we studied carbon chains in which plasmonic resonances are not expected to occur. We used TDDFT to study the emergence of collective resonances in various forms of carbon chains, cumulenes $\mathrm{C}_{n} \mathrm{H}_{4}$, polyynes $\mathrm{C}_{n} \mathrm{H}_{2}$, and alkenes $\mathrm{C}_{n} \mathrm{H}_{n+2}$. The excitation energy and dipole oscillation strengths of these systems were determined through TDDFT by using the TURBOMOLE package. We determined how collective plasmonlike resonances arise from single-electron excitations when the number of electrons increases as the carbon chain lengthens. The collective excitation behavior is then compared with that of metallic atomic chains. Our TDDFT results showed longitudinal collective modes for cumulenes and polyynes, as well as for finite-length chains. These collective excitations exhibit the same behavior as that of longitudinal "plasmon" previously identified in sodium and silver chains, although polyynes are gapped in the long chain limit. Such longitudinal excitations are absent in alkenes. However, unlike metal atomic chains, carbon chains exhibited no transverse collective mode. The band structure of periodic atomic chains was calculated by using the standard local density functional method. These structures were used to interpret the results and to relate the single-electron excitation to the collective plasmonlike response. Within the one-particle quantum-well picture, the longitudinal mode in the linear atomic chain arises from intraband transition with $\Delta q=1$, where $q$ is the quantum number of quantum wells. $\Delta q=1$ intraband transitions can be found in metallic (e.g., Na) chains and in carbon chains (cumulenes and polyynes), such longitudinal collective mode is rather "generic". Meanwhile, the transverse modes of the sodium chains are attributed to interband transitions with an even $\Delta q$ (dominated by $\Delta q=0$ ), and such transverse collective excitations only form if the allowed $\Delta q=0$ transitions occur between bands that are parallel to each other. Such bands can be found in simple metals, but not in carbon chains.
\end{abstract}

DOI: 10.1103/PhysRevB.90.075418

PACS number(s): 72.15.Nj, 71.45.Gm, 73.20.Mf

\section{INTRODUCTION}

Localized surface plasmons in metallic nanostructures have attracted considerable attention in both theoretical and practical studies for their potential applications in optical images [1], optical storages [2], ultrasensitive chemicals, and biological sensing [3-5]. Plasmonic resonance is sustained by the collective oscillations of conducting electrons. Such characteristic oscillation frequency can be tuned by adjusting the size, shape, material, and the surrounding environment of metallic nanoparticles [6]. From the perspective of quantum mechanics, the absorption spectra of metal clusters can be interpreted as transitions between molecular orbitals for clusters with a few electrons. Thus, the manner in which collective oscillations arise from the transitions between Kohn-Sham (KS) orbitals and the number of electrons required to sustain plasmonic-like collective modes are worth investigating

According to linear response theory, the Lehmann representation of the density response function has poles at the excitation energies of the system. For an extended system, the poles with a finite imaginary part should correspond to

\footnotetext{
*ken.ming.lin@gmail.com

†tcleungtw@gmail.com
}

the collective excitation $[7,8]$. In a finite system, identifying which excitation belongs to the collective excitation from the absorption spectra may be difficult. Several researchers have calculated and asserted the existence of plasmon modes in one-dimensional atomic chains after Nilius et al. fabricated gold chains on NiAl [9]. For example, Yan et al. used timedependent density-functional theory (TDDFT) to identify the existence of the longitudinal $(L)$, transverse "end" (TE), and transverse "central" (TC) resonance modes in linear sodium atomic chains. The modes are classified as plasmon collective excitations because the oscillation strengths of the $L$ and TC modes are proportional to the number of atoms [10]. DePrince et al. showed that plasmon modes emerge from a linear hydrogen atomic chain because the charge density oscillates continuously throughout the system [11]. Plasmon resonance is also observed in noble metal atomic chains in the presence of localized $d$ electrons $[12,13]$. Nayyar et al. recently identified the local plasmonic electron oscillations around dopant atoms in transition-metal-doped gold chains [14].

The majority of studies on the plasmonlike resonance of atomic chains have focused on simple and noble metals, which are metallic in the limit of large $N$, where $N$ is the number of atoms. However, the collective response of atomic chains that comprise nonmetallic elements as well as whether these chains sustain similar plasmonlike excitations remain unknown. Our 
study investigates the collective response of atomic chains that become nonmetallic in the long-chain limit. We consider two types of gapped systems: those that arise from Peierls instability [15], wherein the gaps are typically opened at the zone boundary with a "physical coupling" origin (electronphonon coupling), and those with gaps derived from chemical bonding. In particular, we determine whether plasmonlike resonance emerges from carbon chains, including cumulenes $\left(\mathrm{H}_{2} \mathrm{C}_{n} \mathrm{H}_{2}\right)$, polyynes $\left(\mathrm{HC}_{n} \mathrm{H}\right)$, and alkenes $\left(\mathrm{H}\left[\mathrm{CH}_{2}\right]_{n} \mathrm{H}\right)$, and we elucidate how single electron excitations relate to plasmonlike resonance. We verify whether the longitudinal collective excitations in cumulenes and polyynes are similar to those in $\mathrm{Na}$ and $\mathrm{Ag}$, whereas transverse excitations are distinct. Such similarities/differences can be explained by using a one-particle quantum-well picture.

\section{CALCUlations METHOD}

Two types of calculations were performed in this study. The first type is the local density approximation (LDA) [16,17]. In the LDA calculations, the accurate frozen-core full-potential projector augmented-wave (PAW) method was used [18], as implemented in the Vienna ab initio simulation package (VASP) [19-22]. The calculations for structure optimization are based on local density functional theory using the exchange and correlation effects described by generalized gradient approximation (GGA) [23-27]. The atomic chains were placed in a cuboidal supercell with interchain distances equal to at least $10 \AA$. With such a separation, the chain-to-chain interactions and the overlap of wave functions became negligible, such that the chain can be regarded as decoupled entities. The geometric structure of the atomic chains is determined by structure relaxations according to the conjugate gradient method. All forces acting on the atoms are less than $0.02 \mathrm{eV} / \AA$ when the chain is in the equilibrium state. For infinite atomic chains, the length of the unit cell along the chain axis is determined by minimizing the total energy of the system. The $k$-point sampling was set to $1 \times 1 \times 80$ for infinite chains and to $1 \times 1 \times 1$ for finite chains. The second type of calculation is TDDFT, which is used to determine the related properties of excited states and the corresponding oscillator strengths. The excitation energies and oscillation strength were obtained through the linear response formalism in the frequency domain developed by Casida [28]. The excitation energies $\Omega$ are the eigenvalues of the Casida equation as given by

$$
\left[\begin{array}{ll}
A & B \\
B & A
\end{array}\right]\left[\begin{array}{l}
X \\
Y
\end{array}\right]=\Omega\left[\begin{array}{rr}
-1 & 0 \\
0 & 1
\end{array}\right]\left[\begin{array}{l}
X \\
Y
\end{array}\right],
$$

where $A$ and $B$ are the orbital rotation Hessians. The normalization condition of the eigenvectors are given by

$$
\sum_{k=i, a}\left(X_{i, a}^{2}-Y_{i, a}^{2}\right)=1,
$$

where $i$ and $a$ label the occupied and unoccupied orbitals, respectively. The contribution of a single electron excitation from orbital $i$ to orbital $a$ is defined as

$$
\left|C_{i, a}\right|^{2}=X_{i, a}^{2}-Y_{i, a}^{2}
$$

The oscillator strengths are determined as

$$
f_{n}=\frac{2}{3} \sum_{\alpha=1,2,3} \sum_{i, a}\left|x_{i, a}^{\alpha} \omega_{i, a}\left(X_{i, a}-Y_{i, a}\right)\right|^{2} .
$$

In this equation, $\omega_{a, i}=\varepsilon_{a}-\varepsilon_{i}$ denotes the differences between the Kohn-Sham energy eigenvalues and the quantity $x_{i, a}^{\alpha}$ is the position matrix element, given by

$$
x_{i, a}^{\alpha}=\left\langle\phi_{i}\left|x^{\alpha}\right| \phi_{a}\right\rangle, \quad \alpha=1,2,3,
$$

where $\phi_{i}$ and $\phi_{a}$ are the occupied and unoccupied Kohn-Sham eigenfunctions, respectively, and $x^{\alpha}$ is the position operator along the $\alpha$ direction in the Cartesian coordinate.

The exchange-correlation functional in our study is local in time and is adiabatic, which indicates that the exchange correlation functional is the ground-state functional with the density at time $t$. The PBE functional [29-32] and the def2-TZVPP basis $([5 s 4 p 3 d] /[14 s 8 p 3 d],[5 s 3 p 2 d 1 f] /[11 s 6 p 2 d 1 f]$, and $[3 s 2 p 1 d] /[5 s 2 p 1 d]$ for sodium, carbon, and hydrogen, respectively) were implemented in TURBOMOLE V6.2 package, and the ESCF module was used to calculate both the ground and excited states [33-36]. The inner electrons were described by the relative effective core potential. The grids were of m5 quality. With the use of these parameters, the oscillation strength of the allowed dipole transition and the dominant contributions were calculated. TDDFT provides accurate excitation energies for small clusters, whereas LDA results offer a simple one-body picture to understand excitations better.

\section{RESULTS AND DISCUSSION}

Figure 1 shows the calculated oscillator strength of the sodium chains, cumulenes, polyynes, and alkenes as a function
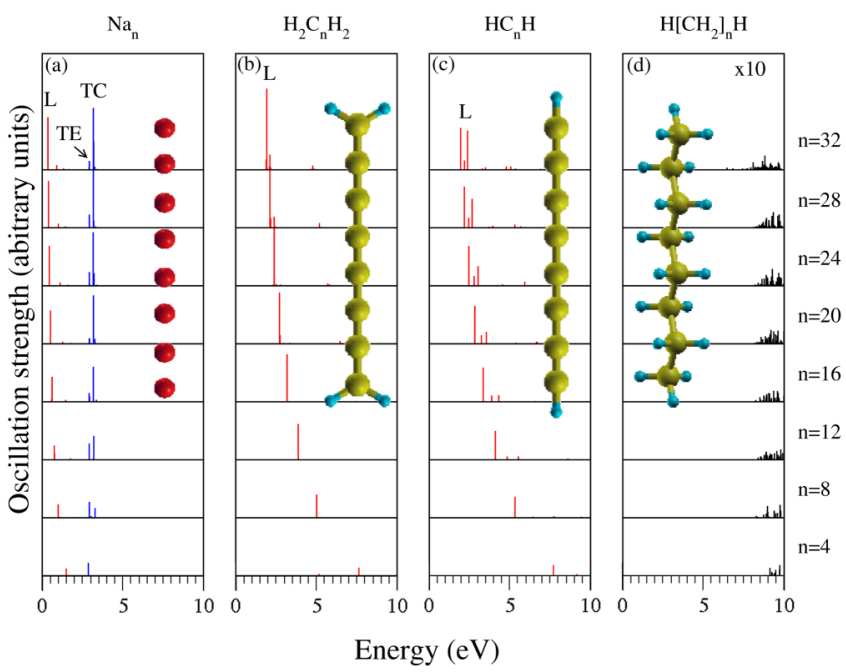

FIG. 1. (Color online) Oscillation strength spectrum calculated by TDDFT for (a) linear sodium chains, (b) cumulenes $\mathrm{H}_{2} \mathrm{C}_{n} \mathrm{H}_{2}$, (c) polyynes $\mathrm{HC}_{n} \mathrm{H}$, and (d) alkenes $\mathrm{H}\left[\mathrm{CH}_{2}\right]_{n} \mathrm{H}$ with a variable number of atoms $N$ in the chain. The longitudinal resonance modes are indicated by $L$ (in red) in sodium, cumulenes, and polyynes chains. Transverse plasmon end and center modes, as indicated by TE and TC (in blue), were observed only in sodium chains. No plasmonlike modes were found in $\mathrm{H}\left[\mathrm{CH}_{2}\right]_{n} \mathrm{H}$. 
of energy for atomic chains containing different numbers of atoms $(N=4,8,12,16,20,24,28$, and 32). The modes labeled by $L$ correspond to the longitudinally polarized dipole excitations. In this case, the charge oscillation runs along the chain length. The TDDFT calculations revealed $L$ modes for sodium chains, cumulenes, and polyynes. The cumulenes have a single $L$ mode with strong oscillator strength, and the behavior of this chain is similar to that of sodium chains. Meanwhile, polyynes have multipeak $L$ modes with strong oscillator strength.

Notably, the increase in oscillator strength versus the number of atoms is a necessary but insufficient condition for defining a collective excitation. This observation is important because any claim for plasmonic response will become meaningless if excitation is not collective. For example, we consider a model system consisting of a collection of $N$ independent noninteracting oscillators, each with a unique resonance frequency that differs from that of others by a very small number. The calculated response of this model system will possess a strong peak of integrated oscillator strength at the resonance frequency. This large peak is actually the superposition of $N$ small peaks, each having a small oscillator strength. Meanwhile, a truly collective excitation should have one large peak possessing large oscillator strength with a linewidth that depends on damping.

If we use a time-propagation TDDFT algorithm and obtain the response through Fourier transform (which has a limited frequency resolution because of the maximum number of time steps used), the response of an $N$-independent-oscillator model system and collective excitation will appear similar, that is, both will exhibit an integrated oscillator strength that increases linearly with $N$.

One feature can help pinpoint a collective excitation involving $N$ particles versus a collection of degenerate independent oscillators. As the number of atom $(N)$ grows, the peak in the oscillator strength spectrum does not shift in frequency if the oscillators are noninteracting. However, for plasmonic collective excitations in a chainlike system, the peak must redshift as $N$ increases because during collective excitation, the current flows from one end of the chain to another. Therefore, the increase in oscillator strength versus the number of atoms, combined with the redshift as the number of atoms increases and the real space picture of current flow, enables the determination of whether the excitation is collective and plasmonlike. The frequency domain approach (Casida formalism) within the TDDFT framework provides additional information on collective excitations. Figure 2 shows the longitudinal mode oscillator strength spectrum with charge oscillators running along the length of the chain for cumulenes and polyynes. The red lines in Figs. 2(a) and 2 (b) indicate the eigenvalues of the Casida equation, whereas the blue lines display the oscillator strength of each eigenstate. Although the number of eigenstates increases as $N$ increases, only a few states show significant oscillator strength.

The sum of the oscillator strength of the dominating states (one or two states for cumulenes and three states for polyynes) increases linearly as $N$ increases, as shown in Fig. 2(c). These states are collective excitations. The oscillation strength increases with increasing chain length because more electrons participate in collective oscillations [10]. Therefore, both short
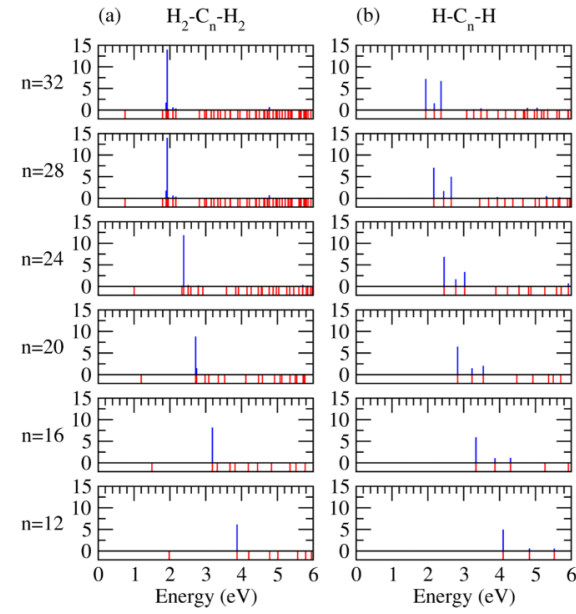

(c)

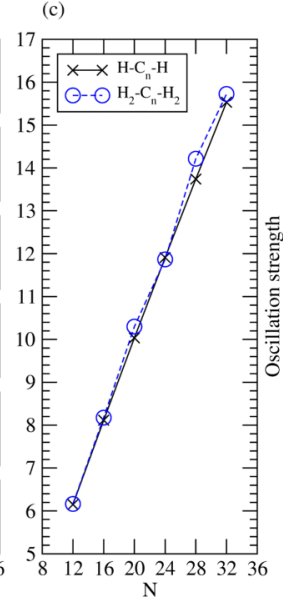

FIG. 2. (Color online) Longitudinal mode oscillator strength spectrum for (a) cumulenes and (b) polyynes. The red lines mark the eigenvalues of the Casida equation, whereas the blue lines display the oscillator strength of each eigenstate. (c) Sum of the oscillator strength of the dominating states.

cumulene and polyyne atomic chains support longitudinal plasmonlike resonances although they are mainly composed of carbon atoms. Notably, polyyne is an insulator in the long-chain limit. However, no $L$ mode exists for alkenes. The broad peaks that appear above $8 \mathrm{eV}$ are transverse excitations, and the strength of the individual peak does not increase with chain length [see Fig. 1(d)].

The transverse modes indicated by subscript $T$ correspond to the transversely polarized dipole excitations, in which the charge oscillations run perpendicular to the length of the chain. Unlike the case of the $L$ mode, $T$ modes are only exhibited by sodium chains. No $T$ modes up to $20 \mathrm{eV}$ were observed for all the carbon chains that we considered. When the number of $\mathrm{Na}$ atoms in the $\mathrm{Na}$ chain exceeded four, the $T$ mode was split into two peaks labeled as TE and TC. Under the TE mode, the charge oscillations were localized at the end of the chain. Meanwhile, the charge oscillation was delocalized near the center of the chain under the TC mode. We found that $\omega_{\mathrm{TE}}<\omega_{\mathrm{TC}}$, which is consistent with the fact that edge modes have lower frequency than their bulk. The intensity of the TE mode was saturated when the number of atoms exceeded 8 . The intensity of the TC mode increased almost linearly with length when $N$ was greater than 8 . The results for the $\mathrm{Na}$ chain are consistent with those found in the earlier real-time TDDFT study by Yan et al. [10].

The plasmonic excitation behavior of finite atomic chains has to be related to the electronic properties of an infinitely long chain. The band structures of infinite linear sodium chains, linear carbon chains, linear dimerized carbon chains, and zigzag carbon chains with two hydrogen atoms per carbon are shown in the left panel of Fig. 3. The interatomic distance for the infinite linear sodium was $3.4 \AA$, whereas that for the linear carbon chains was $1.28 \AA$. The lowest band $(n=1)$ was an $s p_{z} \sigma$ band and the next degenerate bands $(n=2,3)$ were $p_{x}\left(p_{y}\right) \pi$ bands. The $\sigma$ bands of infinitely long linear carbon chains were centered at $-9 \mathrm{eV}$ below the Fermi level and were $7 \mathrm{eV}$ wide. Therefore, these bands are not shown in Fig. 3. For 

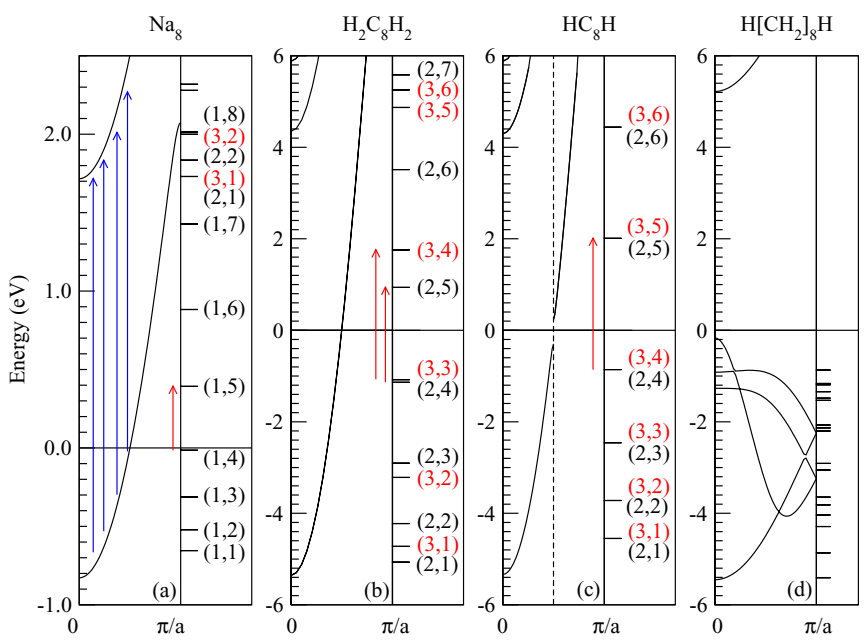

FIG. 3. (Color online) Electronic structure of infinitely long atomic chains (left panel) and finite atomic chains (right panel) for (a) sodium chains, (b) cumulenes, (c) polyynes, and (d) alkenes. The band $(n)$ and quantum-well $(q)$ state indices are labeled as $(n, q)$. The red arrows indicate the transitions that make the dominant contribution to the longitudinal plasmon modes, whereas the blue arrows indicate the dominant transitions for the transverse resonance modes.

the infinitely long dimerized carbon chain, the carbon-carbon distances of 1.254 and $1.314 \AA$ were determined by minimizing the total energy of the system and the bond alternation that resulted from Peierls instability. The band structures of the infinite dimerized linear carbon chain were almost the same as those of the infinite linear carbon chain, except that a $0.5 \mathrm{eV}$ band gap formed at the zone boundary because of the Peierls distortion, as shown in Fig. 3(c). The carbon-carbon distance of the infinite zigzag carbon chain was $1.53 \AA$ with an apical angle of $113.7^{\circ}$. This chain is an insulator with a direct band gap of $5.4 \mathrm{eV}$. The infinite linear, infinite dimerized, and infinite zigzag carbon chain represent the $N \rightarrow \infty$ of cumulenes, polyynes, and alkenes, respectively.

Electron confinement can cause the formation of quantumwell states (QWS) when the size of atomic chains decreases to the nanometer range. The energy of QWS can be described by the quantization condition

$$
2 k(\varepsilon) N a+2 \Phi(\varepsilon)=2 q \pi,
$$

where $k$ is the wave vector along the chain direction of energy $\varepsilon$ in the bulk band, $q$ is the quantum number that corresponds to the number of half-wavelengths that span the well, $N$ is the number of atoms, $a$ is the interatomic distance, and $\Phi(\varepsilon)$ is the phase shift caused by the edge of reflection.

The one-electron orbital energies calculated using the VASP of the finite-length atomic chains are displayed in the right panels of Fig. 3. The vacuum energies of the infinite- and finitelength atomic chains were set to be equal. The orbitals were labeled as $(n, q)$, where $n$ and $q$ are the band and quantum-well state indexes, respectively. Figure 4 shows the $x y$-averaged partial charge distributions along the axial direction $\rho(z)$ for the first 15 states in $\mathrm{Na}_{8}, \mathrm{H}_{2} \mathrm{C}_{8} \mathrm{H}_{2}$, and $\mathrm{HC}_{8} \mathrm{H}$ atomic chains. The solid and open circles mark the $\mathrm{Na}(\mathrm{C})$ and $\mathrm{H}$ atom positions. The value of $q$ for each orbital was determined by the number

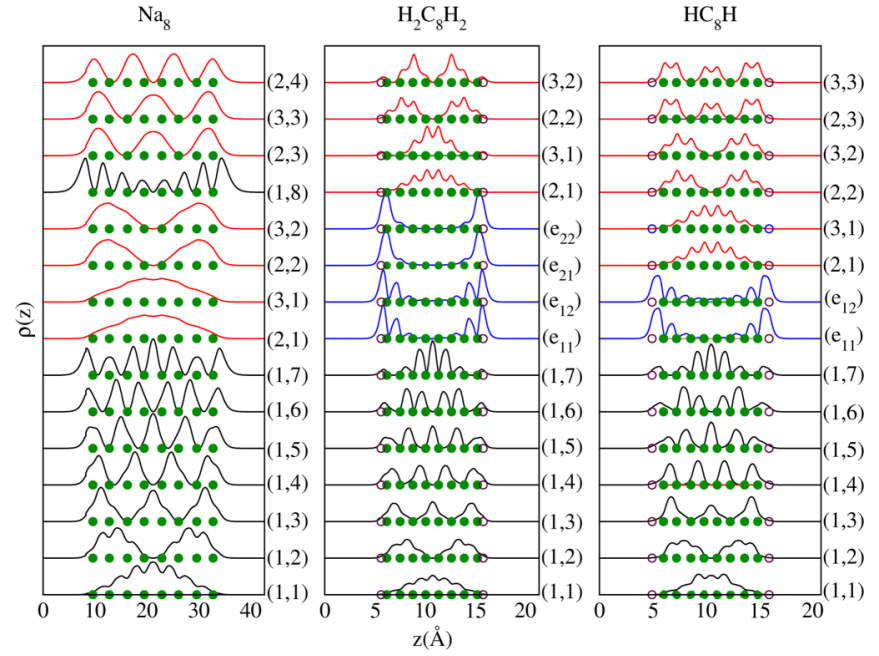

FIG. 4. (Color online) $x y$-averaged partial charge distributions along the axial direction $\rho(z)$ for the first 15 quantum-well states in $\mathrm{Na}_{8}, \mathrm{H}_{2} \mathrm{C}_{8} \mathrm{H}_{2}$, and $\mathrm{HC}_{8} \mathrm{H}$ atomic chains.

of half-wavelengths that span the well. These findings are consistent with the results of the one-dimensional particle-ina-box problem with envelope wave functions that corresponds to standing waves in a potential well. Moreover, the charge density profile for the orbitals with lower energy formed ripples because of the local atomic potential modulation. The ripples for the orbitals with higher energy were not significant because these orbitals have higher kinetic energies. In contrast to the zero wave-function boundary condition at the box boundaries in the particle-in-a-box problem, the electrons can smear out several angstroms as direct consequences of the wave nature of electrons and the finite potential barrier.

For the $\mathrm{Na}_{8}$ atomic chain, eight orbitals that belong to the $\sigma$ band were observed $(n=1)$ with $s$ and $p_{z}$ characteristics, whereas the other orbitals were doubly degenerate orbitals that belong to the QWS in the degenerate $\pi$ band $(n=2,3)$ with $p_{x}$ and $p_{y}$ characteristics. The band gap between the $\sigma$ and $\pi$ bands exhibited edge states for the linear carbon chains. In the case of $\mathrm{HC}_{8} \mathrm{H}$, the first edge state $\left(e_{11}\right)$ exhibited $s$ and $p_{z}$ characteristics, whereas the next edge state $\left(e_{21}\right)$ exhibited $p_{y}$ characteristics. The $x$ and $y$ directions differ from each other in terms of $\mathrm{H}_{2} \mathrm{C}_{8} \mathrm{H}_{2}$, Thus, the degeneracy of the QWS in the degenerated $\pi$ band $(n=2,3)$ was broken, as shown in Fig. 3(c). The wave function of the atomic chain was either an even or odd function of $z$, as shown in Fig. 4. If $q$ is an odd number, the wave functions are even functions of $z$. Meanwhile, when $q$ is an even number, the wave functions are odd functions of $z$. The strength of a particular dipole-allowed transition was determined by using the square of the matrix element: $\left|\left\langle n_{i}, q_{i}\left|P_{z}\right| n_{f}, q_{f}\right\rangle\right|^{2}$ for longitudinal mode and $\left|\left\langle n_{i}, q_{i}\left|P_{x}, P_{y}\right| n_{f}, q_{i}\right\rangle\right|^{2}$ for transverse mode. Therefore, the matrix element is nonvanishing only if $\Delta q$ is odd for longitudinal mode and $\Delta q$ is even for transverse mode.

The description of the QW picture reveals that the primary transitions responsible for the $L$ mode in the $\mathrm{Na}_{8}, \mathrm{H}_{2} \mathrm{C}_{8} \mathrm{H}_{2}$, and $\mathrm{HC}_{8} \mathrm{H}$ atomic chains are the $\Delta n=0$ and $\Delta q=1$ transitions, as indicated schematically in Fig. 3. Although small contributions are made by other transitions such as $\Delta n=$ 

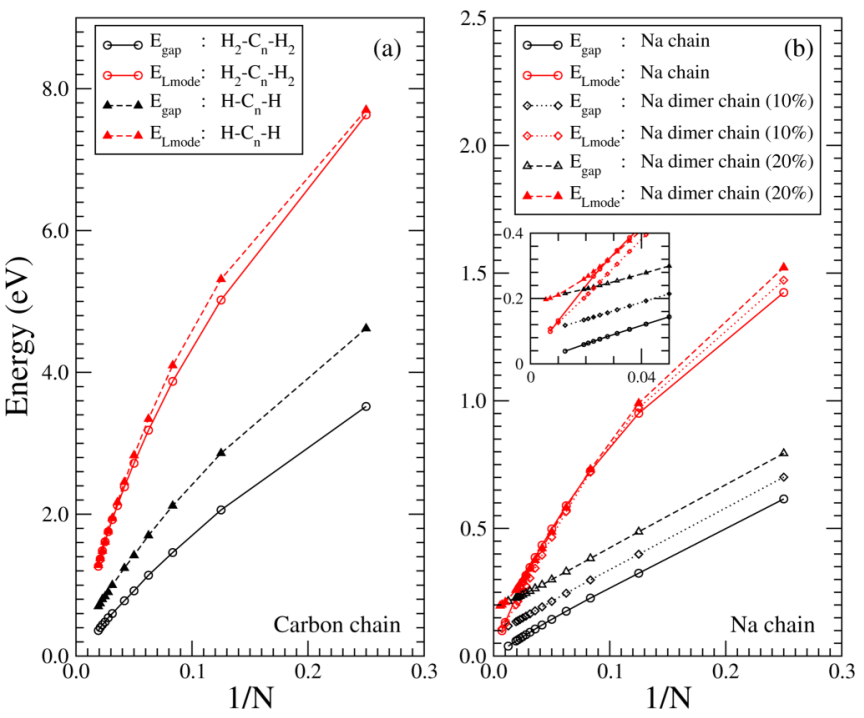

FIG. 5. (Color online) HOMO-LUMO gap $E_{\text {gap }}$ (black) and the first excitation energy of the $L$ mode $E_{L \text { mode }}($ red) as a function of the reciprocal number of carbon atoms $1 / N$ for (a) cumulenes and (b) polyynes in linear and dimerized sodium chains. The solid and dashed lines represent the linear atomic chain and linear dimerized chain, respectively. The inset in panel (b) highlights the large- $N$ limit.

$0, \Delta q=3,5, \ldots$, the intraband transition with $\Delta q=1$ is primarily responsible for the $L$ mode in the linear atomic chain, which seems rather generic. In particular, the contribution from the $\Delta q=1$ transitions in the longitudinal modes was $92.3 \%$ for $(1,4) \rightarrow(1,5)$ in $\mathrm{Na}_{8}, 60.2 \%$ for $(3,3) \rightarrow(3,4)$, and $34 \%$ for $(2,4) \rightarrow(2,5)$ in $\mathrm{H}_{2} \mathrm{C}_{8} \mathrm{H}_{2}$, and $91.2 \%$ for $(2,4),(3,4)$ $\rightarrow(2,5),(3,5)$ in $\mathrm{HC}_{8} \mathrm{H}$, as indicated by the red arrows in Fig. 3. The infinite zigzag carbon chain is an insulator with a direct band gap. Thus, no intraband transition with $\Delta q=1$ was observed in the QWS. This finding explains why the $L$ mode is missing in alkenes. This observation can be extended to atomic chains with different lengths considered in this paper.

We now study the Peierls distortion effect on the plasmon excitation of linear atomic chains. The polyynes exhibit strong bond length alternation, which reflects the alternation between single and triple bonds. By contrast, all carbon-carbon bonds exhibited similar bond lengths in the cumulenes. Figure 5(a) shows the highest occupied molecular orbital-lowest unoccupied molecular orbital (HOMO-LUMO) gap and the first excitation energy of the $L$ mode for polyynes and cumulenes calculated by TURBOMOLE. For $N>8$, the HOMO-LUMO gap of the polyynes and cumulenes varied linearly with the inverse size of the chain. In the limit of infinite chains, the polyynes exhibited a finite HOMO-LUMO gap, whereas the cumulenes were found to become metallic. Nevertheless, although the HOMO-LUMO gaps of the polyynes and cumulenes differed, their first excitation energies were almost the same. To determine whether these results are also true for metal atomic chains, the HOMO-LUMO gaps and the excitation energies of the $L$ mode for dimerized sodium chains with various chain lengths were calculated. The results are displayed in Fig. 5(b). We deliberately set a fairly large bond length alternation to make the dimerization effect easily observable. For the 20\% dimerization, the bond lengths $r_{1}$ and $r_{2}$ of the central bond and the two (equivalent) neighboring bonds in the dimerized chains are given, respectively, by $1.1 \mathrm{a}$ and $0.9 a$, where $a$ is the optimized bond length of the infinite linear sodium chain. We noted that the excitation energies of the $L$ mode for the dimerized sodium chains were also very close to those of sodium chains, although their HOMO-LUMO gaps exhibited significant differences. These results suggest that the excitation energies of the $L$ mode of finite linear atomic chains are only weakly affected by Peierls distortion. The main contributions $\left(\left|C_{i a}\right|^{2}\right)$ for $\Delta q$ equal to 1 and 3 for the longitudinal plasmon mode of cumulenes, polyynes, $\mathrm{Na}$ chains, and 20\% dimerized Na chains are shown in Table I. We found that the $L$ mode arises from the $\Delta q=1$ intraband transition for linear atomic chains with equal bond lengths. By contrast, for dimerized atomic chains, the contributions of the $\Delta q=3$ intraband transition to the $L$ mode increased with the chain length. Although the $L$ mode of the carbon chain has the strongest contribution (in terms of percentage) from the HOMO-LUMO transition, the intraband transition with $\Delta q=3,5, \ldots$ still had a contribution, depending on the whole structure of the valence band. The similarity of the structure of the valence band of the linear dimerized carbon chain to that of the linear carbon chain except for a small band gap opening at the Fermi level produced similar excitation energies for their $L$ modes, as shown in Fig. 3.

In the following section, we will investigate why the transverse $(T)$ plasmon modes found in metal atomic chains were absent in linear carbon chains. As previously mentioned, the strength of a particular transition is determined by the square of the matrix element. Within the QW picture, the matrix element is nonvanishing only if $\Delta q$ is even for the $T$ mode because of the symmetry of the quantum-well states. The upper two panels of Fig. 6 show the square of the matrix element, $m_{x}^{2}=\left|\left\langle n_{i}, q_{i}\left|P_{x}\right| n_{f}, q_{f}\right\rangle\right|^{2}$, from the LDA calculations of the sodium chains and polyynes as a function of transition energy $\omega_{a i}$ for $\Delta q=0,2$, and 4. Notably, the matrix element is dominated by $\Delta q=0$ transitions. Up to 12 allowed transitions were within the energy range $(2.34 \mathrm{eV}$,

TABLE I. Dominating contribution $\left(\left|C_{i a}\right|^{2}\right)$ for $\Delta q$ equal to 1 and 3 of the $L$ mode of cumulenes, polyynes, Na chains, and dimerized Na chains for different numbers of atoms $N$.

\begin{tabular}{|c|c|c|c|c|c|c|c|c|}
\hline & \multicolumn{2}{|c|}{ Cumulenes } & \multicolumn{2}{|c|}{ Polyynes } & \multicolumn{2}{|c|}{ Na chains } & \multicolumn{2}{|c|}{ Dimerized Na chains } \\
\hline & $\Delta q=1$ & $\Delta q=3$ & $\Delta q=1$ & $\Delta q=3$ & $\Delta q=1$ & $\Delta q=3$ & $\Delta q=1$ & $\Delta q=3$ \\
\hline$N=8$ & 0.94 & 0.01 & 0.91 & 0.06 & 0.99 & 0.06 & 0.77 & 0.16 \\
\hline$N=16$ & 0.95 & 0.02 & 0.81 & 0.10 & 0.99 & 0.09 & 0.56 & 0.43 \\
\hline$N=24$ & 0.93 & 0.02 & 0.70 & 0.16 & 0.98 & 0.16 & 0.57 & 0.42 \\
\hline
\end{tabular}



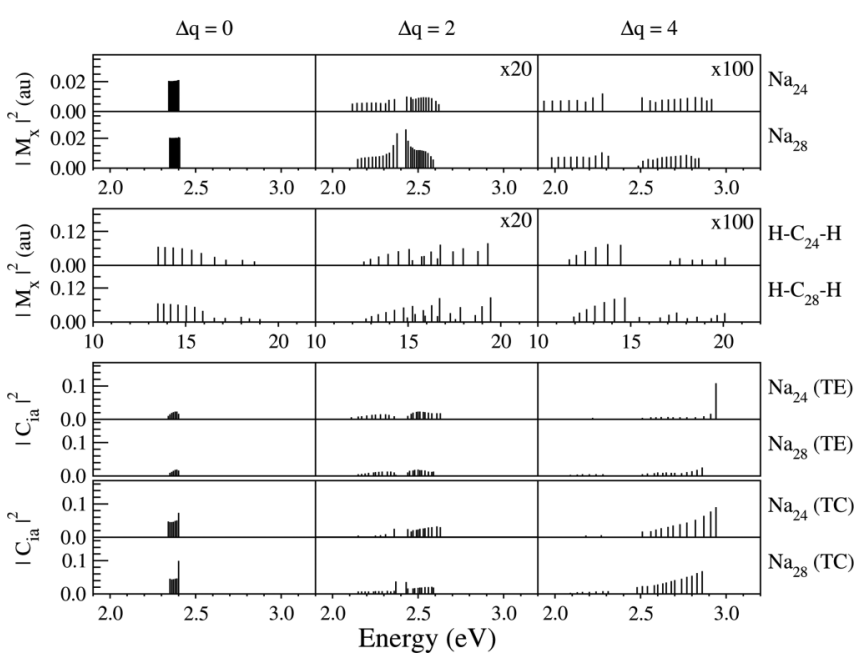

FIG. 6. Square of momentum matrix element $\left|M_{x}\right|^{2}$ vs transition energy for various $\Delta q$ of sodium chains and polyynes with $N$ equal to 24 and to 28 , as shown in the upper two panels. Dominating contribution $\left(\left|C_{i a}\right|^{2}\right)$ vs transition energy for various $\Delta q$ of the $T$ mode of sodium chains with $N$ equal to 24 and 28, as shown in the lower two panels.

$2.40 \mathrm{eV}$ ) for $\mathrm{Na}_{24}$, and 14 allowed transitions in the range $(2.34 \mathrm{eV}, 2.41 \mathrm{eV})$ for $\mathrm{Na}_{28}$. The transitions that can be characterized as interband transitions were within the oneparticle band picture $\left(s p_{z} \sigma\right.$ band $\rightarrow p_{x}, p_{y} \pi$ band), i.e., $(1,1) \rightarrow(2,1),(3,1) ;(1,2) \rightarrow(2,2),(3,2) ; \ldots ;(1, N / 2) \rightarrow$ $(2, N / 2),(3, N / 2)$. For polyynes, 11 transitions were within the range $(13.5 \mathrm{eV}, 18.7 \mathrm{eV})$ for $\mathrm{HC}_{24} \mathrm{H}$ and 13 transitions were within the range $(13.5 \mathrm{eV}, 19.0 \mathrm{eV})$ for $\mathrm{HC}_{28} \mathrm{H}$, which can be characterized as interband transitions $\left(s p_{z} \sigma\right.$ band $\rightarrow p_{x}, p_{y}$ $\pi$ band), as shown in Fig. 6(b). The dominant contribution transition $\left|C_{i a}\right|^{2}$ that corresponds to the $T$ mode of the sodium chains on the basis of the TDDFT calculations is shown in Fig. 6(c). Interestingly, all allowed transitions contributed to $T$-mode excitation. Our results indicate that the $T$ mode of the linear atomic chain is a collective excitation that involves all possible symmetry-allowed transitions.

From the left panel of Fig. 6(a), we note that the width of the spectrum for the $\Delta q=0$ transitions in sodium chains was only approximately $0.1 \mathrm{eV}$ because the lower band (the band that crosses the Fermi level) and the second band are almost parallel to each other. This feature is characteristic of free-electron-like systems confined to one dimension. This finding indicates that the transition energies of all allowed transitions are very close to one another, and can therefore accumulate to form a collective excitation. By contrast, the width of the spectrum for the $\Delta q=0$ transitions in polyynes was approximately $5.5 \mathrm{eV}$ because the first band, which is a degenerate $\sigma$-band of the $s$ and $p_{z}$ character, has dispersions that are very different from those of the second and third bands, which are the $\pi$ bands of mixed $p_{x}$ and $p_{y}$ characters. This finding suggests that the transition energies of the allowed transition are very different from one another, such that they do not easily form a collective excitation, which results in the absence of the transverse plasmon mode in polyynes.

\section{CONCLUSION}

This study provides clear evidence that the plasmonlike resonance of the $L$ mode emerges from carbon chains, i.e., the hydrogen-terminated cumulenes and polyynes, on the basis of TDDFT numerical computations. However, cumulenes and polyynes, unlike metal atomic chains, have no $T$ modes. Furthermore, no plasmon resonance occurs in alkenes. With the quantum-well picture, the primary transitions responsible for the longitudinal mode in a linear atomic chain are intraband transitions with $\Delta q$ equal to 1 . Although the HOMO-LUMO gaps of the polyynes and cumulenes are significantly different, their first excitation energies are almost the same. The excitation energies of the longitudinal modes of finite linear atomic chains are only weakly affected by the Peierls-type bond length alternation. Meanwhile, the transverse modes of the sodium chains are dominated by interband transitions with $\Delta q$ equal to 0 and 2, which results in a collective excitation that involves all the selection rule allowed transitions. The band theory approach outlined in this study can facilitate a qualitative understanding of the plasmonlike excitations of other atomic chain systems.

\section{ACKNOWLEDGMENTS}

We thank Steven Louie for comments and discussions. K.M.L. also thanks Michitoshi Hayashi, Min-Wei Chen, and JianHao Li for helpful discussions. This work is supported by the National Science Council (NSC) of Taiwan under Grant No. NSC 99-2112-M-194-010-MY3 and the National Center of Theoretical Sciences (NCTS South) through the computational Material Research Focus Group, and by the Research Grant Council of Hong Kong through AoE/P02/12. We thank the National Center for High-performance Computing for providing the CPU time.
[1] N. Fang, H. Lee, C. Sun, and X. Zhang, Science 308, 534 (2005).

[2] D. P. Tsai and W. C. Lin, Appl. Phys. Lett. 77, 1413 (2000).

[3] A. M. Kalsin, M. Fialkowski, M. Paszewski, S. K. Smoukov, K. J. M. Bishop, and B. A. Grzybowski, Science 312, 420 (2006).

[4] L. R. Hirsch, R. J. Stafford, J. A. Bankson, S. R. Sershen, B. Rivera, R. E. Price, J. D. Hazle, N. J. Halas, and J. L. West, Proc. Natl. Acad. Sci. (USA) 100, 13549 (2003).

[5] X. Huang, I. H. El-Sayed, W. Qian, and M. A. El-Sayed, J. Am. Chem. Soc. 128, 2115 (2006).
[6] U. Kreibig and M. Vollmer, Optical Properties of Metal Clusters (Springer-Verlag, Berlin, 1995).

[7] A. L. Fetter and J. D. Walecka, Quantum Theory of ManyParticle System (Dover, New York, 2003).

[8] C. A. Ullrich, Time-Dependent Density-Functional Theory (Oxford, New York, 2012).

[9] N. Nilius, T. M. Wallis, and W. Ho, Science 297, 1853 (2002).

[10] J. Yan, Z. Yuan, and S. Gao, Phys. Rev. Lett. 98, 216602 (2007). 
[11] A. E. DePrince, M. Pelton, J. R. Guest, and S. K. Gray, Phys. Rev. Lett. 107, 196806 (2011).

[12] J. Yan and S. Gao, Phys. Rev. B 78, 235413 (2008).

[13] K.-E. Lian, P. Salek, M. Jin, and D. Ding, J. Chem. Phys. 130, 174701 (2009).

[14] N. Nayyar, V. Turkowski, and T. S. Rahman, Phys. Rev. Lett. 109, 157404 (2012).

[15] R. E. Peierls, Quantum Theory of Solid (Oxford University Press, Oxford, 2001).

[16] P. Hohenberg and W. Kohn, Phys. Rev. 136, B864 (1964).

[17] W. Kohn and L. J. Sham, Phys. Rev. 140, A1133 (1965).

[18] P. E. Blöchl, Phys. Rev. B 50, 17953 (1994).

[19] G. Kresse and J. Hafner, Phys. Rev. B 47, 558 (1993).

[20] G. Kresse and J. Hafner, Phys. Rev. B 48, 13115 (1993).

[21] G. Kresse and J. Hafner, Phys. Rev. B 49, 14251 (1994).

[22] G. Kresse and J. Furthmüller, Comput. Mater. Sci. 6, 15 (1996).

[23] J. P. Perdew and A. Zunger, Phys. Rev. B 23, 5048 (1981).

[24] J. P. Perdew and Y. Wang, Phys. Rev. B 33, 8800 (1986).
[25] J. P. Perdew, Phys. Rev. B 33, 8822 (1986).

[26] D. C. Langreth and J. P. Perdew, Phys. Rev. B 21, 5469 (1980).

[27] D. C. Langreth and M. J. Mehl, Phys. Rev. B 28, 1809 (1983).

[28] M. Casida, in Recent Advances in Density Functional Methods, Part I, edited by P. Chong (World Scientific, Singapore, 1995), p. 155.

[29] P. A. M. Dirac, Proc. R. Soc. London, Ser. A 123, 714 (1929).

[30] J. C. Slater, Phys. Rev. 81, 385 (1951).

[31] J. P. Perdew and Y. Wang, Phys. Rev. B 45, 13244 (1992).

[32] J. P. Perdew, K. Burke, and M. Ernzerhof, Phys. Rev. Lett. 77, 3865 (1996).

[33] R. Ahlrichs, M. Baer, M. Haeser, H. Horn, and C. Koelmel, Chem. Phys. Lett. 162, 165 (1989).

[34] O. Treutler and R. Ahlrichs, J. Chem. Phys. 102, 346 (1995).

[35] M. V. Arnim and R. Ahlrichs, J. Comp. Chem. 19, 1746 (1998).

[36] R. Bauernschmitt and R. Ahlrichs, Chem. Phys. Lett. 256, 454 (1996). 